\title{
Forschendes Lehren und Lernen - ein mehrdimensionales Modell für die Lehrpraxis aus Perspektive der empirischen Wissenschaften
}

\section{Natascha Selje-Aßmann}

Ausgangspunkt des Beitrags ist die Analyse von Definitionen des Forschenden Lernens im Hinblick auf Herausforderungen bei der praktischen Umsetzung in empirischen Wissenschaften. Die vorliegenden Konzepte werden um die Perspektive eines mehrdimensionalen Modells ergänzt, das unterschiedliche Phasen und Grade des Forschungsprozesses integriert. Das Modell lässt sich zur Reflexion von Formaten Forschenden Lernens bei der Planung einzelner Lehrveranstaltungen oder zur Integration von Forschendem Lernen entlang eines Curriculums einsetzen.

\section{Definitionen und Modelle des Forschenden Lernens und ihre Herausforderungen}

Forschendes Lernen ist in der Hochschullehre ein häufig verwendetes Schlagwort. So vielfältig wie die darunter entwickelten Formate sind auch die Ansätze, Forschendes Lernen zu definieren. Das Ringen um die Begrifflichkeit führt zu einer zunehmenden Differenzierung in Termini wie Forschungsbezogene Lehre (Rueß, Gess \& Deicke, 2016), Forschungsgeleitetes, -begleitendes, -basiertes oder -orientiertes Lernen unter dem Überbegriff des Forschungsnahen Lehrens und Lernens (Huber, 2014). Zudem haben sich verwandte Konzepte wie Problem- oder Fallorientiertes, Projektbasiertes und Situiertes Lernen (Reinmann, 2009) etabliert. 
Für Lehrende der Fachwissenschaften sind sowohl konzeptionelle Abgrenzungen als auch Konsequenzen für die didaktische Umsetzung nicht immer ersichtlich. Abweichungen in Perzeption und praktischer Durchführung von Forschung in den Disziplinen verstärken die Kontroverse. Im Folgenden werden Konzepte zu Forschendem Lernen und Herausforderungen diskutiert, die bei der Implementierung in empirischen Wissenschaften auftreten können.

Bei der Komposition der Begriffe „Forschen" und „Lernen“ werden unterschiedliche Schwerpunkte gesetzt. Wird der Aspekt des Lernens fokussiert, beschreibt Forschendes Lernen einen Lernstil, der sich durch neugieriges, problemorientiertes und kritisches Denken sowie autonomes und kreatives Arbeiten auszeichnet (Multrus, 2012) und dem Vertiefen von Inhalten oder Methoden des Faches dient (siehe Typus Lernen; Rueß, Gess \& Deicke, 2016). Bei Definitionen, die den Aspekt des Forschens betonen, tritt das Erlernen von Fachwissen in den Hintergrund, während das Durchlaufen bzw. Nachvollziehen des Forschungsprozesses eine übergeordnete Rolle spielt (siehe Typus Forschen; Rueß, Gess \& Deicke, 2016; Huber, 2009; Reiber, 2012; Wildt, 2009). Bei diesen am Forschungsprozess orientierten Definitionen umfasst Forschendes Lernen die selbstständige Wahl des Themas, der Strategie und Methoden, Recherchen, das Risiko von Irrtümern und Umwegen sowie die Chance für Zufallsfunde und Unerwartetes, den Anspruch an Wissenschaftlichkeit sowie die Prüfung und Darstellung der Ergebnisse inklusive deren Nachvollziehbarkeit und Relevanz (BAK, 1970). Ziel ist,

„konkrete Forschungserfahrungen zu sammeln und den Prozess der Erkenntnisgewinnung bzw. Wissensprüfung zu reflektieren, um mit der wissenschaftlichen Arbeitsund Denkweise vertraut zu werden" (Messner, 2003, zitiert nach Tremp, 2005, S. 344).

Die hohe Komplexität von Forschendem Lernen erfordert dabei von den Lernenden Wissen, Fertigkeiten und Fähigkeiten, die sie noch nicht oder nur teilweise besitzen (Gotzen, Beyerlin \& Gels, 2015). Zur Generierung wissenschaftlicher Erkenntnisse benötigte Kompetenzen sollen einerseits durch Forschendes Lernen entwickelt werden, werden andererseits aber vorausgesetzt. Die Fähigkeit zu Forschen - definiert als systematische, regelgeleitete wissenschaftliche Praxis der Erkenntnisgewinnung und Bearbeitung von Problemstellungen (Fichten, 2012) - umfasst eine Bandbreite an Kompetenzen, einschließlich der Kenntnis der normativen Regeln der jeweiligen Disziplin. Der Anspruch an Wissenschaftlichkeit und damit einhergehende Konventionen wie Nachvollziehbarkeit und Reproduzierbarkeit setzen dem Prozess enge Grenzen. Die Entwicklung einer Forschungsfrage erfordert neben fundiertem Grundlagenwissen die Kenntnis des aktuellen Wissenstands in der Detailfrage. Zur exakten Versuchsplanung und Wahl einer angemessenen 
Erhebungs- und Auswertungsmethodik müssen Kenntnisse in Statistik und/oder über verfügbare fachspezifische Methoden vorhanden sein (Selje-Aßmann et al., 2017). Der Zeitbedarf für Forschendes Lernen erhöht sich zudem, wenn Fertigkeiten im Umgang mit Geräten, Prozessen und Software erworben werden müssen. Hinzu kommen fachübergreifende forschungsmethodische Kenntnisse, z. B. zu Literaturrecherche oder wissenschaftlichem Schreiben, Fähigkeiten in Zeit- und Projektmanagement oder Teamarbeit. Dies würde bedeuten, dass Studierende empirischer Wissenschaften erst ab einem gewissen Umfang an methodischen Fertigkeiten und Kenntnissen zu Forschendem Lernen befähigt sind. Die Kompetenzen werden im Verlauf einer Forschungslaufbahn über Studium, Bachelor- und Masterarbeit, Promotion und Postdoc-Phase sukzessive entwickelt. Der Prozess erfordert Erfahrung, Wiederholung und Reflexion und übersteigt meist die zeitliche Dimension einzelner Lehrveranstaltungen. Forschendes Lernen in sequenzielle Untereinheiten zu unterteilen (siehe Etappen im Zürcher Framework, Tremp \& Hildbrand, 2012) erlaubt, einzelne Schritte des Forschungsprozesses wiederkehrend und mit gesteigerten Anforderungen zu üben. Modelle zu Forschendem Lernen sollten diesen Entwicklungsprozess einbeziehen.

In vielen Definitionen ist die Selbstständigkeit der Lernenden unverzichtbarer Bestandteil von Forschendem Lernen (siehe das Selbstständigkeitspostulat bei Fichten, 2012). Andere heben die Selbständigkeit von Lernenden bei der Wahl von Kontext und Fragestellung als das entscheidende Merkmal hervor, das Forschendes Lernen von verwandten Konzepten wie Anwendungs- oder Problembasiertes Lernen unterscheidet (Wildt, 2011). Damit wären im Rahmen von Drittmittelprojekten ausgeschriebene Abschlussarbeiten keine Formate Forschenden Lernens. Die Frage ist, ob das dieser Definition von Forschendem Lernen zugrunde liegende Ideal des freien Forschens anhand selbstständig entwickelter Fragestellungen in Zeiten von Drittmittelabhängigkeit, teurer Ausstattung, langen Begutachtungsdauern von Forschungsanträgen und des Rufs nach gesellschaftlicher Relevanz der Forschungsrealität entspricht. Bei Huber klingt eine andere Perspektive an: Forschendes Lernen als Teilhabe an Wissenschaft als einem immer offenen Prozess, im Zusammenwirken und -lernen von Lehrenden und Lernenden, an Problemen arbeitend, an denen diese interessiert und bei denen sie auch emotional engagiert sind, nach Erkenntnissen suchend, die auch für Dritte interessant sind. Die Studierenden sollen das ihnen jeweils größtmögliche Maß an Selbstständigkeit, eigener Aktivität und Kooperation mit anderen realisieren können (Huber, 2013). Entsprechend kann Forschendes Lernen als Beteiligung der Studierenden an der Forschung von Lehrenden verstanden bzw. in der Praxis durchgeführt werden. Die selbstständige Themenwahl kann damit nicht das entscheidende Merkmal zur Abgrenzung des Konzeptes darstellen, entscheidender ist die aktive Rolle der Lernenden in einem Forschungsprozess. 
Viele Modelle zu Forschendem Lernen leiten sich von der zweidimensionalen Matrix von Healey mit den Kategorien „Inhaltlicher Fokus“ (Forschungsergebnisse vs. -prozess) und „Partizipationsgrad der Studierenden“ (partizipierend vs. rezipierend, bzw. lernenden- vs. lehrendenzentriert) ab (Healey, 2005). Wenige und starre Kategorien können aber die Komplexität, Variabilität und Prozesshaftigkeit von Forschendem Lernen nicht ausreichend darstellen. Der Ansatz, akademische Lehre innerhalb dieser Matrix durch zusätzliche Ebenen wie inhaltliche Tiefe oder Breite, angeleitetes oder selbstorganisiertes Lernen aufzufächern, zeigt, dass weitere Dimensionen benötigt werden, um die Bandbreite von Forschendem Lernen abzubilden (Reinmann, 2014). Auch die Matrix von Rueß, Gess und Deicke wurde um die inhaltliche Unterkategorie „Forschungsmethoden“ und bezüglich des Aktivitätsniveaus der Studierenden um die Unterkategorie „anwendend“ ergänzt (Rueß, Gess \& Deicke, 2016). Da Methoden Mittel zum Zweck darstellen, ist die Frage, inwieweit Ergebnisse ohne das methodische Vorgehen der Literaturrecherche aufgearbeitet werden können bzw. ob die Anwendung von fachspezifischen Methoden ohne Fragestellung möglich ist.

Des Weiteren erzeugt die Dimension des Partizipationsgrades der Studierenden eine Polarität zwischen Lehrenden- und Lernendenperspektive. In Folge der Abkehr von der vermeintlich lehrendenzentrierten Vorlesung führt dies in vielen Konzepten zu einer Beschränkung auf die Lernendenperspektive. Lehrende sind jedoch zentrale Figuren bei der Gestaltung der Hochschullehre, im Besonderen im interaktiven Lehr-Lern-Prozess der Beteiligung Studierender an der eigenen Forschung. Im Doppelradmodell von Lübcke, Reinmann und Heudorfer wird die Lehrperson als Gestalterin der Mikroebene von Lehrveranstaltungen zwar dargestellt, steht der Lernendenebene jedoch mehr oder weniger unverbunden gegenüber (Lübcke, Reinmann \& Heudorfer, 2017). Dagegen kann kollaboratives Forschen als gemeinsamer Entwicklungsprozess von Lehrenden und Lernenden mit gleicher Blickrichtung betrachtet werden.

\section{Das mehrdimensionale Modell für Forschendes Lehren und Lernen}

Um die Aspekte verschiedener Zielsetzungen, der sequenziellen Entwicklung von Kompetenzen, unterschiedlicher Grade der Selbstständigkeit von Lernenden und der Interaktionen mit Lehrenden aufzugreifen, wurde ein mehrdimensionales Modell entwickelt. Es basiert auf Erfahrungen mit studentischen Forschungsprojekten, die in empirischen Wissenschaften auf dem Forschungsfeld der betreuenden Lehrenden durchgeführt wurden. 
Für die zugrunde liegende Definition von Forschendem Lernen werden Lernhandlung (durch die Lernenden), Lerninhalt (Nahziel der Lehrveranstaltung) und das übergeordnete Fernziel der universitären Bildung (Langemeyer, 2017) beleuchtet. Die Lernhandlung, Lernen im Format der Forschung (Wildt, 2009), beschreibt den Weg des Erkenntnis- oder Kompetenzerwerbs durch Interaktion mit dem Forschungsgegenstand, wie Messungen, Beobachtungen, Recherche, Befragungen. Der Lerninhalt kann den Erwerb von vertieftem Fachwissen, (forschungs-)methodischen Fertigkeiten sowie persönlichen und sozialen Kompetenzen umfassen. Damit verknüpft ist die Frage, wozu Lehre im Format der Forschung (Ludwig, 2011) an der Hochschule eingesetzt wird. Fernziel der universitären Bildung ist die intellektuelle Bildung durch Wissenschaft, die wissenschaftlich basierte Beschäftigungsbefähigung und Persönlichkeitsentwicklung der Studierenden (Wissenschaftsrat, 2000), charakterisiert durch eine forschende und verantwortliche Grundhaltung, einen dynamischen Wissensbegriff und Lernen als kontinuierlicher, reflexiver Prozess der Erkenntnis (Brinckmann et al., 2002). Die Einheit von Lehre und Forschung sollte sich nicht nur in der Lehrperson manifestieren, sondern auch auf Ebene der Lernenden realisiert werden. Um den Fokus nicht auf den Lernprozess zu beschränken, lassen sich zusätzlich Ziele aus der Perspektive der Lehrenden und der Forschungsgemeinschaft definieren. Im Idealfall gehen die Generierung originärer Daten und neuer Erkenntnisse sowie die Rekrutierung von wissenschaftlichem Nachwuchs aus dem Forschungsprozess hervor.

Zusammenfassend liegt dem Modell ein Verständnis von Forschendem Lernen als einer (inter-)aktiven Form des Lernens zugrunde, bei der Lernende Fachwissen, methodische Fertigkeiten und forschungsmethodische Kompetenzen durch systematisches Vorgehen und Interaktion mit anderen Forschenden erwerben, mit dem Anspruch von Wissenschaftlichkeit und dem Ziel der Professionalisierung durch Wissenschaft. Forschendes Lernen wird als Kompetenz verstanden, die in einem fortschreitenden Prozess entwickelt wird, aber der Förderung und Anleitung bedarf (Multrus, 2012).

In der Hochschullehre wird dieser Prozess durch Interaktion mit Lehrenden geprägt, weshalb Forschendes Lehren und Lernen gemeinsam gedacht wird. Im Vordergrund steht weniger die Ausdifferenzierung von Formaten - das Modell visualisiert vielmehr die Beziehungen von Lernenden, Lehrenden und Lerninhalt sowie den Umfang und wissenschaftlichen Anspruch in Kontexten von Forschendem Lernen. Diese können unterschiedliche Ausprägungen mit fließenden Übergängen annehmen und werden daher in Form von Achsen dargestellt. Die Verortung eines Lehrformates entlang der Achsen lässt Rückschlüsse zu, z. B. auf die benötigte Betreuungsform, den Zeitaufwand oder das Prüfungsformat. Der Bildungsstand der Lernenden kann hingegen bestimmen, welcher inhaltliche Schwerpunkt oder welcher Grad an Selbstständigkeit verwirklicht werden kann. Das Modell soll helfen, bei der Studiengangsplanung Formate zunehmender Selbstständigkeit der Lernenden sowie unterstützende Angebote 
zum Üben einzelner Teilschritte des Forschungsprozesses zu entwickeln. Es enthält folgende Dimensionen, die im Weiteren näher erläutert werden: 1. den Lerninhalt, 2. die (thematische) Vorstrukturierung, 3. die Art und Intensität der Betreuung, 4. den zeitlichen und thematischen Umfang und 5. den wissenschaftlichen Anspruch.

\subsection{Der Lerninhalt}

Wie in anderen Modellen mit dichotomer Einteilung (Healey, 2005) kann der Fokus entweder stärker auf dem Erwerb von Forschungskompetenzen oder auf dem Erwerb fachwissenschaftlicher Erkenntnisse liegen (Abb. 1). Beim „Forschen Lernen“ steht das Erlernen forschungsmethodischer Kompetenzen im Vordergrund, die in einem vollständigen Forschungszyklus erfahren oder teilweise, auch in Seminaren oder Projekten, erarbeitet werden können. Unterstützende Formate zum „Handwerk der Wissenschaft" - mit Inhalten wie Versuchsdesign, Präsentationstechniken, gute wissenschaftliche Praxis - sind auf der linken Seite der Achse einzuordnen. Beim „Lernenden Forschen" kann der Forschungsprozess das Format sein, Wissen zu erarbeiten oder Erkenntnisse zu generieren, ohne selbst Lerninhalt zu sein. Bei der Planung solcher Formate wird jedoch deutlich, dass in der Praxis diese Aspekte nicht voneinander zu trennen sind und es im Gegenteil sinnvoll erscheint, bei der Erforschung einer fachlichen Fragestellung anhand der eigenen Forschungstätigkeit den Forschungsprozess zu reflektieren. Idealfall und Stärke des Forschenden Lehrens und Lernens liegen in der engen Verknüpfung beider Aspekte und damit in der Mitte der Achse.

\section{Dimension: Inhaltlicher Fokus, Lernziel, Methodik}

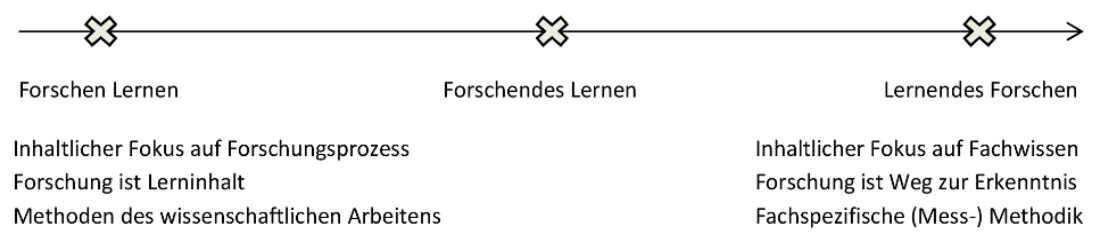

Abb. 1 Die Dimension des Nahziels eines Formates Forschenden Lehrens und Lernens thematisiert den Fokus des Lerninhaltes und die dazugehörige Methodik

Die Verortung des Lehrformates entlang dieser Dimension ist ausschlaggebend für die detaillierte thematische Planung sowie Inhalt und Format einer möglichen Prüfung. Bei der Einbettung von Forschendem Lehren und Lernen in ein 
Curriculum ist es sinnvoll, neben der Vermittlung von fachlichen Grundlagen mit Formaten zu beginnen, die den Forschungsprozess reflektieren oder Teile des Prozesses üben. Darauf aufbauend kann der fachliche Inhalt fokussiert werden, das heißt sich auf der Achse immer weiter nach rechts zu bewegen. Als Klimax haben die Lernenden den Forschungsprozess verinnerlicht und können sich forschend auf die Generierung neuer Erkenntnisse konzentrieren. Forschungskompetenzen können durch Methoden der Selbstreflexion geprüft werden, bei denen zum Beispiel die Qualität der gewählten Referenzen oder Vorschläge für zukünftige Forschungsansätze bewertet werden. Liegt der Fokus auf Fachwissen, treten inhaltliche und methodische Kenntnisse in den Vordergrund. In der Verknüpfung der Lerninhalte sind Prüfungen in Form gängiger wissenschaftlicher Formate - wie Vortrag, Poster oder Abschlussarbeit - zur Präsentation der Forschungsleistung und Diskussion sowohl des Vorgehens als auch der Ergebnisse zweckmäßig.

\section{2 (Thematische) Vorstrukturierung}

Die (thematische) Vorstrukturierung ist besonders in der Planungs- und Anfangsphase bedeutend und beleuchtet, inwieweit eine spezifische Fragestellung und das Forschungsdesign durch die Lernenden entwickelt werden können bzw. welche Vorgaben und Planungen vonseiten der Lehrenden nötig sind. Die Verortung in dieser Dimension hängt von Vorkenntnissen der Lernenden und Rahmenbedingungen des Fachgebietes oder Curriculums ab und kann sich auf die Motivation und die emotionale Involviertheit der Lernenden sowie die Organisation und Realisierbarkeit des Formates durch die Lehrenden auswirken.

2. Dimension: Vorstrukturierung, Selbstständigkeit bei der Entwicklung der Fragestellung

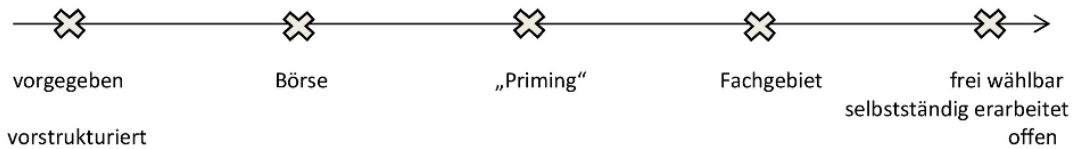

Abb. 2 Dimension der (thematischen) Vorstrukturierung bzw. Selbstständigkeit bei der Entwicklung der spezifischen Forschungsfrage bei der Gestaltung von Formaten Forschenden Lehrens und Lernens

Bei der Entwicklung der Forschungsfrage nimmt die Beziehung zwischen Lernendem und Lerninhalt unterschiedliche Ausprägungen entlang einer Achse zunehmender 
Selbstständigkeit an und wird aufseiten der Lehrenden durch einen abnehmenden Grad der Vorstrukturierung kontrastiert. Gegenpole sind eine durch die Lehrenden vorgegebene Problemstellung und eine durch die Lernenden entwickelte Forschungsfrage (Abb. 2). Zwischenschritte sind die Wahl einer Fragestellung aus einem Angebot möglicher Themen („Börse“), die Formulierung einer Forschungsfrage nach Eingrenzung des Themenspektrums anhand von Literatur, durch eine einleitende Vorlesung oder durch den Forschungsschwerpunkt des Fachbereichs (hier als „Priming“ bezeichnet).

Selbstbestimmtheit und Verfolgen eigener Interessen gelten als wichtige Bedingungen für die Motivation und die Qualität des Lernens (Deci \& Ryan, 1993). Dies spricht für eine von den Lernenden gewählte Fragestellung. Die Zielrichtung von Formaten Forschenden Lehrens und Lernens sollte deshalb im Verlauf des Curriculums in Richtung zunehmender Selbstständigkeit weisen. Doch ist zu berücksichtigen, dass Studierende zu Beginn des Studiums mit dem Selbstständigkeitsanspruch oft überfordert sind. Nach eigener Erfahrung können sie hohe Motivation auch durch einen eigenen Beitrag zu einem größeren Forschungsprojekt infolge sozialer Eingebundenheit erleben (siehe dazu ebenfalls Deci \& Ryan, 1993) - im Gegensatz zur Generierung von Daten in standardisierten Praktika, die über das Üben der Datengenerierung hinaus keinen weiteren Nutzen aufweisen. Zusätzlich erhöht ein durch den Forschungsschwerpunkt der Lehrenden vorgegebenes Thema deren Begeisterung für die Fragestellung, was sich ebenfalls positiv auf die Lehr-Lern-Situation auswirken kann. Diese Perspektive der Lehrenden fehlt in den auf die Perspektive der Lernenden gerichteten Ansätzen. Betreuende, die über eine hohe Expertise auf ihrem Forschungsgebiet verfügen, können neben einem tiefen Verständnis für ihr Fach anhand der geteilten Leidenschaft den Spaß am Forschen vermitteln und als Vorbilder gute wissenschaftliche Praxis vorleben.

Die Verortung in dieser Dimension wird von der Verortung innerhalb der Dimension des Lerninhaltes beeinflusst. Steht der Erwerb von forschungsmethodischen Kompetenzen - wie das Erstellen von Hypothesen oder eines wissenschaftlichen Textes - im Vordergrund, kann die Bearbeitung einer eigenständigen Fragestellung leicht verwirklicht werden. Je stärker der Inhalt auf die fachliche Seite rückt und praktische Datenerhebungen erfordert, desto schwerer ist die freie Themenwahl realisierbar und von der Finanzierung und Ausstattung laufender Forschungsprojekte sowie von vorhandenen Kompetenzen zur Betreuung abhängig.

\subsection{Art und Intensität der Betreuung}

Die Bedeutung der sozialen Interaktion von Lehrenden und Lernenden für den Lernerfolg (Hattie, 2009; Schneider \& Preckel, 2017) wird in einigen Definitionen 
zu Forschendem Lernen thematisiert (Huber, 2013; Multrus, 2012; Reiber, 2012). Ergebnisse der Lernforschung legen nahe, dass unerfahrene Lernende in neuen Lernsituationen genaue Anleitungen benötigen bzw. der Lernzuwachs höher ausfällt, wenn sie intensiv betreut werden (Kirschner, Sweller \& Clark, 2006; Mayer, 2004). Eine den Fähigkeiten und Bedürfnissen der Lernenden angepasste Anleitung ist gerade bei Forschendem Lehren und Lernen zur Vermeidung von Überforderung und Frustration essenziell. Die Intensität der Betreuung, die Beziehung zwischen Lehrenden und Lernenden, wird deshalb in einer eigenen Dimension thematisiert und betrifft vorwiegend die Phase der praktischen Durchführung.

\section{Dimension: Intensität der Betreuung, Verhältnis Lehrende und Lernende}

\begin{tabular}{|c|c|c|}
\hline Stark angeleitet & Betreuung entsprechend Bedarf & $\begin{array}{r}\text { selbstständiges Arbeiten, } \\
\text { Teamarbeit }\end{array}$ \\
\hline \multicolumn{3}{|c|}{ Rolle der Lehrenden } \\
\hline anleitend/lehrend & coachend/beratend & kollegial \\
\hline
\end{tabular}

Abb.3 Dimension der Betreuungsintensität mit wechselnden Rollen der Lehrenden bei der Durchführung von Formaten Forschenden Lehrens und Lernens

Die Dimension reicht von stark angeleitetem bis zum selbstständigem Arbeiten (Abb. 3) und ist unabhängig von der (thematischen) Vorstrukturierung. Die Bearbeitung eines frei oder in einer Börse gewählten Themas kann aufgrund geringer Vorkenntnisse bei gleichzeitig komplexer Methodik eine intensive Anleitung erfordern. Wird hingegen ein vorgegebenes Thema selbstständig bearbeitet, kann sich die Betreuung auf die Bewertung der Ergebnisse beschränken (Abb. 4). Im Verlauf des Studiums sollte die Betreuungsintensität schrittweise reduziert werden, um Studierende zu Beginn nicht zu überfordern, aber die Entwicklung einer eigenständigen Forschungspersönlichkeit zu fördern. Zielrichtung ist erneut die zunehmende Selbstständigkeit bzw. Entwicklung der Fähigkeit zur Kollaboration in einem Forschungsteam. Aufseiten der Lehrenden bedarf es eines konvergenten Prozesses mit Wechsel im eigenen Rollenverständnis. Je nach Vorwissen und Persönlichkeit der Lernenden können Lehrende betreuende, anleitende, beratende, Vorbild- oder kollegiale Funktionen übernehmen. Die Betreuungsintensität wirkt sich auf das Zeitbudget der Lehrenden, die mögliche Anzahl an Teilnehmenden und die Anforderungen, die an die Lernenden gestellt werden, aus. Die Grenzen der Betreuung und der Grad der geforderten Selbstständigkeit sollten gegenüber den 
Lernenden expliziert werden. Konfliktpotenzial ergibt sich bei der Integration von Forschendem Lehren und Lernen in die Forschungstätigkeit der Lehrenden. Wenn diese am Ergebnis des Forschungsprozesses interessiert sind, kann es zu einer stärkeren Anleitung als notwendig kommen, um die Qualität der Ergebnisse zu sichern.
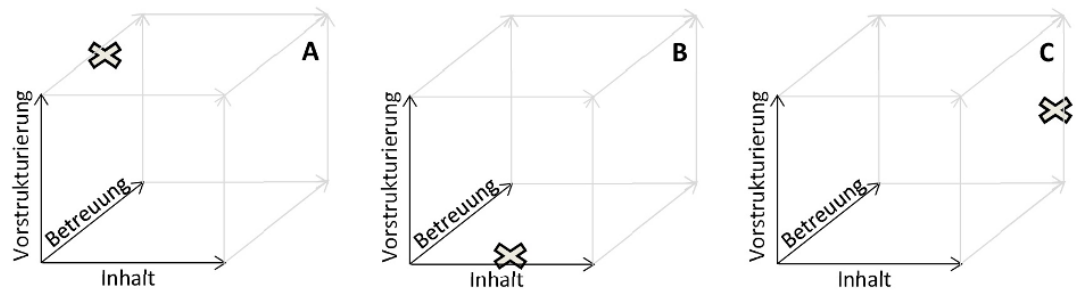

A) Wissenschaftliches Schreiben mit eigener Themenwahl und mittlerer Betreuungsleistung

B) Erforschung vorgegebener Fragestellung mit Reflexion des Forschungsprozesses und intensiver Betreuung

C) Erforschung eingegrenzter Fragestellung mit Fokus auf Forschungsergebnissen und geringer Betreuung

Abb. 4 Beispiele für die Verortung von Formaten Forschenden Lehrens und Lernens in den kombinierten Dimensionen Lerninhalt, (thematische) Vorstrukturierung und Betreuung (zu beachten ist, dass die beiden Dimensionen Vorstrukturierung und Betreuung ihren höchsten Wert im Achsenschnittpunkt aufweisen und in Richtung zunehmender Selbstständigkeit der Lernenden weisen)

\subsection{Zeitlicher und thematischer Umfang}

Aufgrund der Komplexität und Individualität im Vergleich zu standardisierten Veranstaltungen ist Forschendes Lehren und Lernen ein zeitintensives Format für Lernende wie Lehrende. Der inhaltliche Umfang (Tiefe oder Breite) und die benötigte Zeit für ein studentisches Forschungsprojekt können stark variieren und bedingen sich gegenseitig; dies wird über das unterschiedliche Volumen des Lernraums dargestellt (Abb. 5). So können bei inhaltlichem Fokus auf den Forschungsprozess einzelne Teilschritte thematisiert oder der gesamte Forschungsprozess kann durchlaufen werden. Bei inhaltlichem Fokus auf Fachwissen erfordern kleine, in sich geschlossene Fragestellungen oder die Beschränkung auf einen Teilaspekt - wie die Präzision einer Messmethode - einen geringeren Zeitaufwand als ein komplexer Versuchsansatz. Wichtige Aspekte - wie der Umgang mit Rückschlägen, die mit dem Verwerfen von Hypothesen, fehlgeschlagenen Messungen oder dem Ändern 
von Versuchsansätzen einhergehen - machen Formate Forschenden Lehrens und Lernens langwieriger und schwerer planbar als standardisierte Versuche. Umgekehrt wirkt sich das zeitliche Limit einer Lehrveranstaltung auf den realisierbaren Inhalt aus und kann eine Beschränkung auf Teilaspekte des Forschungsprozesses oder durchführbare Experimente erfordern.

\section{Dimension: Zeitlicher und inhaltlicher Umfang}
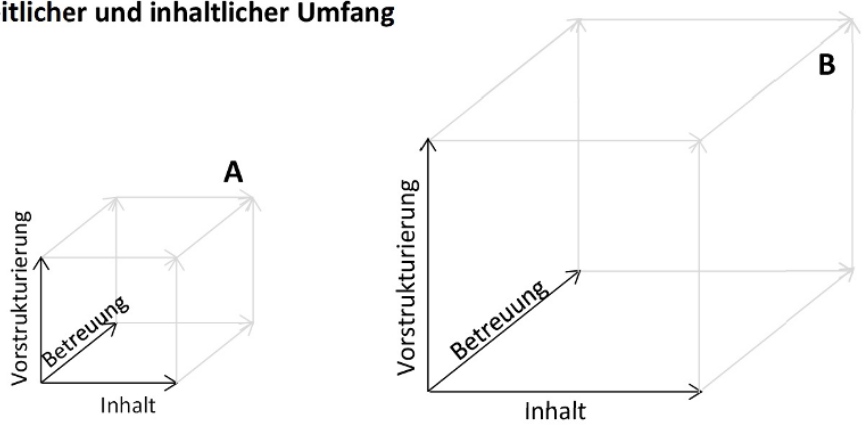

A) Geringer zeitlicher oder inhaltlicher Umfang ermöglicht Bearbeitung von Teilschritten des Forschungsprozesses bzw. einfacher Fragestellungen

B) Längere Zeiträume ermöglichen inhaltliche Tiefe oder Breite, komplexe Fragestellungen bzw. das Durchlaufen des gesamten Forschungsprozesses

Abb. 5 Dimension des zeitlichen und inhaltlichen Umfangs von Formaten Forschenden Lehrens und Lernens

\subsection{Wissenschaftlicher Anspruch}

Der Erwerb von Forschungskompetenz wird als iterativer Entwicklungsprozess mit unterschiedlichen Niveaus betrachtet, den es für einen optimalen Lernzuwachs auf jeder Stufe bedarfsgerecht zu unterstützen gilt. Die Ansprüche an Forschendes Lehren und Lernen steigern sich mit dem Studienverlauf und den Forschungserfahrungen. Die in Abbildung 6 dargestellte Dimension des wissenschaftlichen Anspruchs integriert alle vorangegangenen Dimensionen insofern, als der wissenschaftliche Anspruch auf den jeweiligen Erfahrungen, Kenntnissen und Fertigkeiten aufbaut und ein größerer zeitlicher Umfang eine zunehmende Komplexität erlaubt. Die Dimension thematisiert die Beziehung der Lernenden zur Fachgesellschaft und weist in Richtung des (Fern-)Ziels, der Enkulturation in der jeweiligen Wissenschaftsgemeinschaft (Langemeyer, 2017). Der wissenschaftliche Anspruch wird beispielsweise abgebildet durch das Forum einer möglichen Veröffentlichung der 
Forschungsarbeit: von einer internen Präsentation über eine Präsentation auf einer studentischen Tagung bis hin zu einer Veröffentlichung in einer Fachzeitschrift.

\section{Dimension: Wissenschaftlicher Anspruch, Beziehung zu Fachgesellschaft}

$\begin{array}{lll}\text { gering } & \text { Forum für Veröffentlichung } & \text { Fachpublikum } \\ & \text { nationale/internationale } & \text { Fachtagung } \\ \text { Arbeits-/ } & \text { Konferenz für studentische } & \text { Fachzeitschrift } \\ \text { Peergruppe } & \text { Forschung } & \text { hoch }\end{array}$

Abb. 6 Dimension des wissenschaftlichen Anspruchs mit Auswirkung auf das Forum der Ergebnispräsentation

\subsection{Abschlussbetrachtung}

Dem mehrdimensionalen Modell liegt eine Definition von Forschendem Lehren und Lernen als eine Lehr- und Lernform mit hohem Aktivitätsgrad der Lernenden und expliziter Interaktion mit forschend Lehrenden zugrunde. Anders als die Matrix von Healey (2005) enthält es nicht die Dimension des Aktivitätsgrades der Lernenden. Dieser fließt in die Dimensionen der Vorstrukturierung und der Betreuungsintensität ein.-

Eine zunehmende Selbstständigkeit in der Planung und Durchführung von Forschung geht mit einer wachsenden aktiven Rolle der Lernenden einher und kann abweichend von anderen Definitionen (z. B. Wildt, 2011) graduelle Unterschiede annehmen. Innerhalb des mehrdimensionalen Lernraumes kann Forschendes Lehren und Lernen an jedem beliebigen Punkt angesiedelt werden und unterschiedliche Ausprägungen annehmen (Abb. 7). Die Integration mehrerer Dimensionen erlaubt es, zusätzliche Aspekte von Forschendem Lehren und Lernen zu beleuchten. Durch die Möglichkeit, den Forschungsprozess und/oder den thematischen Inhalt zu fokussieren, lassen sich Fachdisziplinen mit abweichendem Verständnis von „Forschung“ und "Methodik" subsumieren. Methoden werden nicht unabhängig vom Inhalt betrachtet, sondern in fachspezifische (Mess-)Methoden (Kenntnis und praktische Durchführung) und forschungsmethodische Kompetenzen (z. B. sich selbstständig in neue Methoden einarbeiten zu können) unterschieden. Ein zentraler Aspekt ist die übergreifende Entwicklungsrichtung mit dem Ziel der "Forschungskompetenz" bzw. „Ausbildung einer forschenden Grundhaltung“. Der mehrdimensionale Raum 


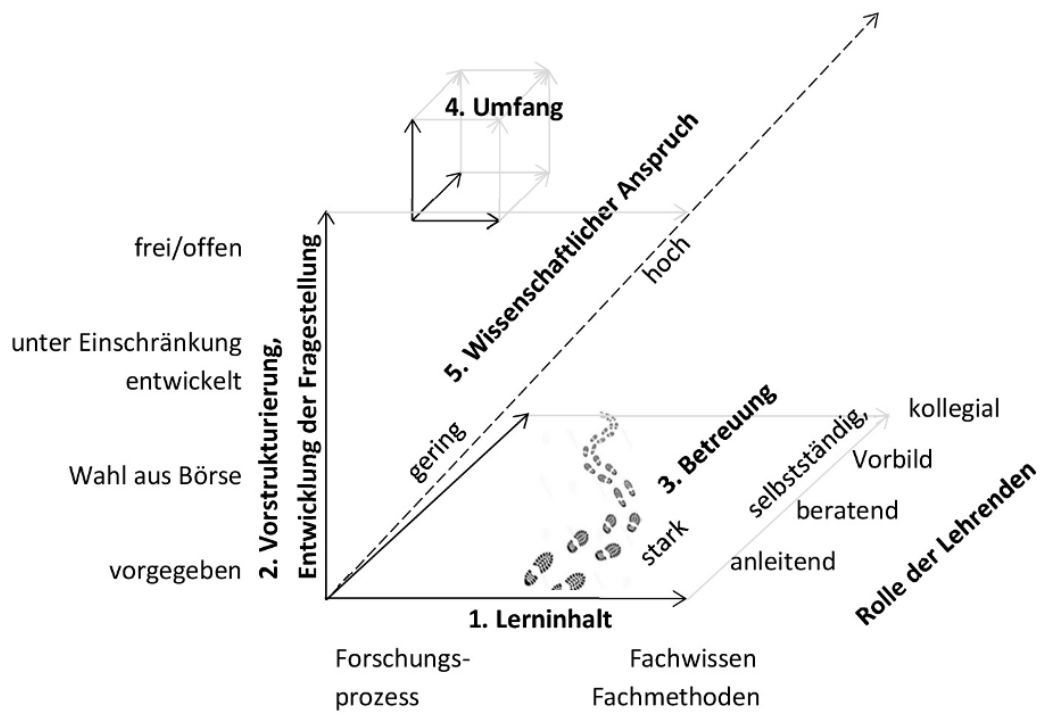

Abb. 7 Das mehrdimensionale Modell zu Forschendem Lehren und Lernen mit den Dimensionen Lerninhalt, (thematische) Vorstrukturierung, Betreuungsintensität, Umfang und wissenschaftlicher Anspruch sowie der Forschungskompetenz bzw. einer forschenden Grundhaltung als übergeordnetes Bildungsziel

verdeutlicht, dass es unterschiedliche Pfade gibt, entlang derer Forschungskompetenz entwickelt werden kann, und dass die Entwicklung einen längeren Prozess erfordert, der schwer innerhalb einer einzelnen Lehrveranstaltung abgedeckt, aber durch sequenzielles Addieren einzelner Wegstrecken erreicht werden kann. Die Freiheitsgrade, die das Modell bei der Einordnung bietet, lassen keine einfachen kausalen Ableitungen zu, sondern erfordern von Lehrenden eine verantwortungsvolle Reflexion des eigenen Formates, um den Begriff von Forschendem Lehren und Lernen nicht zu verwässern. Wichtig bleibt dabei die übergeordnete Zielrichtung „Bildung durch Wissenschaft". Projekte Forschenden Lehrens und Lernens sind durch das Voranschreiten der Wissenschaft und der eigenen Forschung sowie individuelle Kombinationen von lehrend Forschenden und forschend Lernenden einzigartig. $\mathrm{Zu}$ starre Vorgaben können sich negativ auf die intrinsische Motivation bei Lernenden wie Lehrenden auswirken. Bei einem zu starken Fokus auf den Forschungsprozess besteht die Gefahr, dass das fachliche Thema, eigentlicher Kristallisationspunkt des gemeinsamen Interesses, in den Hintergrund gerät. Befürchtet wird, dass unscharfe 
Konturen die Unterscheidung zu verwandten Konzepten unmöglich und das Konzept von Forschendem Lernen überflüssig machen würden (Reinmann, 2009). Doch ermöglicht vielleicht gerade diese Unsicherheit die Freiheit, kreativ verschiedene Spielformen auszuprobieren und den jeweiligen fachspezifischen Besonderheiten und forschenden Persönlichkeiten anzupassen.

\section{Literatur}

Brinckmann, H., Garcia, O., Gruschka, A., Lenhardt, G. \& ZurLippe, R. (2002). Die Einheit von Forschung und Lehre: Über die Zukunft der Universität. Wetzlar: Büchse der Pandora.

Bundesassistentenkonferenz (BAK) (1970). Forschendes Lernen - Wissenschaftliches Prüfen (Schriften der BAK, 5. Neudruck). Bielefeld: UVW.

Deci, E. L. \& Ryan, R. M. (1993). Die Selbstbestimmungstheorie der Motivation und ihre Bedeutung für die Pädagogik. Zeitschrift für Pädagogik, 39, S. 223-238.

Fichten, W. (2012). Über die Umsetzung und Gestaltung Forschenden Lernens im Lehramtsstudium Verschriftlichung eines Vortrags auf der Veranstaltung „Modelle Forschenden Lernens" in der Bielefeld School of Education. Verfügbar unter https://uol.de/fileadmin/ user_upload/diz/download/Publikationen/Lehrerbildung_Online/Fichten_01_2013_Forschendes_Lernen.pdf [09.08.2019].

Gotzen, S., Beyerlin, S. \& Gels, A. (2015). „Steckbrief: Forschendes Lernen“ der TH Köln. Verfügbar unter https://www.th-koeln.de/mam/downloads/deutsch/hochschule/profil/ lehre/steckbrief_forschendes_lernen.pdf [09.08.2019].

Hattie, J. (2009). Visible learning: A synthesis of over 800 meta-analyses relating to achievement. New York: Routledge.

Healy, M. (2005). Linking research and teaching: disciplinary spaces. In R. Barnett (Hrsg.), Reshaping the university: new relationships between research, scholarship and teaching (S. 30-42). Maidenhead, McGraw-Hill: Open University Press.

Huber, L. (2009). Warum Forschendes Lernen nötig und möglich ist. In L. Huber, J. Hellmer \& F. Schneider (Hrsg.), Forschendes Lernen im Studium: Aktuelle Konzepte und Erfahrungen (S. 9-35). Bielefeld: UVW.

Huber, L. (2013). Methodische Anregungen für den Umgang mit pragmatischen Schwierigkeiten im Forschenden Lernen. In L. Huber, M. Kröger \& H. Schelhowe (Hrsg.), Forschendes Lernen als Profilmerkmal einer Universität: Beispiele aus der Universität Bremen (S. 248-255). Bielefeld: UVW.

Huber, L. (2014). Forschungsbasiertes, Forschungsorientiertes, Forschendes Lernen: Alles dasselbe? Das Hochschulwesen (1 \& 2), S.22-29.

Kirschner, P., Sweller, J. \& Clark, R. (2006). Why minimal guidance during instruction does not work: An analysis of the failure of constructivist, discovery, problem-based, experiential, and inquiry-based teaching, Educational Psychologist, 41(2), S.75-86. Verfügbar unter http://www.cogtech.usc.edu/publications/kirschner_Sweller_Clark.pdf [09.08.2019]. 
Langemeyer, I. (2017). Das forschungsbezogene Studium als Enkulturation in Wissenschaft. In H. A. Mieg \& J. Lehmann (Hrsg.), Forschendes Lernen: Wie die Lehre in Universität und Fachhochschule erneuert werden kann (S.91-100). Frankfurt: Campus.

Lübcke, E., Reinmann, G. \& Heudorfer, A. 2017. Entwicklung eines Instruments zur Analyse Forschenden Lernens. Zeitschrift für Hochschulentwicklung, 12(3), S. 191-216. Verfügbar unter https://www.zfhe.at/index.php/zfhe/article/view/1020 [09.08.2019].

Ludwig, J. (2011). Forschungsbasierte Lehre als Lehre im Format der Forschung. Brandenburgische Beiträge zur Hochschuldidaktik, 3, S. 7-18. Universitätsverlag Potsdam. Verfügbar unter http://pub.ub.uni-potsdam.de/volltexte/2011/4985 [09.08.2019].

Mayer, R. (2004). Should there be a three-strikes rule against pure discovery learning? The case for guided methods of instruction. American Psychologist, 59, S. 14-19.

Messner, H. (2003). Erziehungswissenschaften in der Praxis: forschendes Lernen bei pädagogischen Abschlussarbeiten. In Fachhochschule Aargau/Nordwestschweiz (Hrsg.), Kontext: Pädagogik (S. 16-18).

Multrus, F. (2012). Forschung und Praxis im Studium. Befunde aus Studierendensurvey und Studienqualitätsmonitor. Konstanzer Online-Publikations-System (KOPS). Verfügbar unter http://kops.uni-konstanz.de/bitstream/handle/123456789/22246/Multrus_222461. pdf? sequence $=2$ \&isAllowed $=y$ [09.08.2019].

Reiber, K. (2012). Forschendes Lernen im Zeichen von Bologna. In B. Kossek \& C. Zwiauer (Hrsg.), Universität in Zeiten von Bologna: Zur Theorie und Praxis von Lehr- und Lernkulturen (S. 111-119). Vienna University Press.

Reinmann, G. (2009). Wie praktisch ist die Universität? Vom situierten zum Forschenden Lernen mit digitalen Medien. In L. Huber, J. Hellmer \& F. Schneider (Hrsg.), Forschendes Lernen im Studium: Aktuelle Konzepte und Erfahrungen (S.36-52). Bielefeld: UVW.

Reinmann, G. (2014). Hochschuldidaktik: Friedrichshafener Vorträge. Verfügbar unter http:// gabi-reinmann.de/wp-content/uploads/2013/05/Hochschuldidaktik_Friedrichshafener-Vortr\%C3\%A4ge.pdf [09.08.2019].

Rueß, J., Gess, C. \& Deicke, W. (2016). Forschendes Lernen und forschungsbezogene Lehre - empirisch gestützte Systematisierung des Forschungsbezugs hochschulischer Lehre. Zeitschrift für Hochschulentwicklung, 11(2), S.23-44. Verfügbar unter https://www.zfhe. at/index.php/zfhe/article/view/914 [09.08.2019].

Schneider, M. \& Preckel, F. (2017). Variables associated with achievement in higher education: A systematic review of meta-analyses. Psychological Bulletin. Verfügbar unter https:// www.researchgate.net/publication/311789042_Variables_Associated_With_Achievement_in_Higher_Education_A_Systematic_Review_of_Meta-Analyses [09.08.2019].

Selje-Aßmann, N., Poll, C., Tisler, M., Gerstenberg, J., Blum, M. \& Fleischer, J. (2017). Forschendes Lernen in Bachelor- und Masterstudiengängen der Lebenswissenschaften. In H. A. Mieg \& J. Lehmann (Hrsg.), Forschendes Lernen: Wie die Lehre in Universität und Fachhochschule erneuert werden kann (S. 200-209). Frankfurt: Campus.

Tremp, P. (2005). Verknüpfung von Lehre und Forschung: Eine universitäre Tradition als didaktische Herausforderung - In Beiträge zur Lehrerinnen- und Lehrerbildung 23(3), S.339-348. Verfügbar unter https://www.pedocs.de/volltexte/2017/13579/pdf/ BZL_2005_3_339_348.pdf [09.08.2019].

Tremp, P. \& Hildbrand, T. (2012). Forschungsorientiertes Studium - universitäre Lehre: Das „Zürcher Framework“ zur Verknüpfung von Lehre und Forschung. In T. Brinker \& P. Tremp (Hrsg.), Einführung in die Studiengangentwicklung (Blickpunkt Hochschuldidaktik 122, S. 101-116). Bielefeld: Bertelsmann. 
Wildt, J. (2009). Forschendes Lernen: Lernen im „Format“ der Forschung. Journal Hochschuldidaktik, 20(2), S. 4-7.

Wildt, J. (2011). Kompetenzorientertes Prüfen - Eine hochschuldidaktische Sicht. Vortrag in Erlangen am 22.07.2011. Verfügbar unter https://www.hrk-nexus.de/uploads/media/ Tagung-nexus-Kompetenzorientiertes_Pruefen-Wildt_01.pdf [09.08.2019].

Wissenschaftsrat (WR) (2000). Empfehlungen zur Einführung neuer Studienstrukturen und -abschlüsse (Drs. 4418/00). Berlin.

\section{Danksagung}

Das Modell wurde im Rahmen der Koordinations- und Lehrtätigkeit innerhalb des Qualitätspakt-Lehre-Projekts „Humboldt reloaded - Wissenschaftspraxis von Anfang an" an der Universität Hohenheim entwickelt und durch zahlreiche Diskussionen innerhalb des Projektteams geprägt. Wertvolle Kommentare und Anregungen im Besonderen von Dr. Barbara Engler, Dr. Cornelia Frank, Julia Gerstenberg, Dr. Hanna Gölz, Julia Höhn, Annegret Höpner, Rosario Pires, Dr. Christian Poll, Dr. Evelyn Reinmuth und Prof. Martin Blum haben zur Verbesserung dieses Artikels beigetragen.

\section{Zur Autorin}

Dr. rer. nat. Natascha Selje-Aßmann, Dipl.-Biol., ist Koordinatorin des Qualitätspakt-Lehre-Projekts „Humboldt reloaded - Wissenschaftspraxis von Anfang an“ an der Universität Hohenheim und freut sich über Kommentare und Anregungen zur Weiterentwicklung des Modells. E-Mail: n.seljeassmann@uni-hohenheim.de

Open Access Dieses Kapitel wird unter der Creative Commons Namensnennung 4.0 International Lizenz (http://creativecommons.org/licenses/by/4.0/deed.de) veröffentlicht, welche die Nutzung, Vervielfältigung, Bearbeitung, Verbreitung und Wiedergabe in jeglichem Medium und Format erlaubt, sofern Sie den/die ursprünglichen Autor(en) und die Quelle ordnungsgemäß nennen, einen Link zur Creative Commons Lizenz beifügen und angeben, ob Änderungen vorgenommen wurden.

Die in diesem Kapitel enthaltenen Bilder und sonstiges Drittmaterial unterliegen ebenfalls der genannten Creative Commons Lizenz, sofern sich aus der Abbildungslegende nichts anderes ergibt. Sofern das betreffende Material nicht unter der genannten Creative Commons Lizenz steht und die betreffende Handlung nicht nach gesetzlichen Vorschriften erlaubt ist, ist für die oben aufgeführten Weiterverwendungen des Materials die Einwilligung des jeweiligen Rechteinhabers einzuholen. 